\title{
BETTI NUMBERS OF ALEXANDROV SPACES
}

\author{
LIANG-KHOON KOH
}

(Communicated by Christopher Croke)

\begin{abstract}
We obtain a uniform bound on the sum of Betti numbers for a class of complete Alexandrov spaces with narrow ends.
\end{abstract}

\section{INTRODUCTION}

Alexandrov spaces are complete length spaces with a lower curvature bound and finite Hausdorff dimension. One of the essential differences between Alexandrov spaces and Riemannian manifolds is that points in a compact set do not have a uniform lower bound on their contractibility radius near a singular point.

Gromov gave an upper bound for the Betti numbers of a compact Riemannian manifold in terms of its diameter and the lower bound of the sectional curvatures (see [2]). This estimate in particular shows that most manifolds admit no metrics of nonnegative sectional curvature. Specifically, in the class of Riemannian manifolds with lower sectional curvature bound $K_{M} \geq k \quad(k \leq 0)$ and upper diameter bound $\operatorname{diam} M \leq d$, Gromov proved that the sum of Betti numbers is bounded above,

$$
\sum_{k=0}^{n} b_{k}(M) \leq C(n)^{1+\sqrt{-k d}}
$$

with respect to any field $F$, where $C(n)$ is a constant depending only on $n=$ $\operatorname{dim} M$.

The estimate for the upper bound of the sum of Betti numbers does not hold if instead of $K_{M} \geq k$ one assumes that the Ricci curvature $\operatorname{Ric} \geq(n-1) k$. This can be seen by examples of Sha and Yang (see $[6,7])$. In this case, however, one can still prove that the first Betti number of $M$ (with respect to the field of real numbers) satisfies $b_{1}(M) \leq C(n)^{1+\sqrt{-k d}}$ (see [3]).

For the case of open Riemannian manifolds, Shen introduced the concept of width for a complete Riemannian manifold and gave an upper estimate of the sum of Betti numbers for the class of thin Riemannian manifolds (see [8]).

Let $M$ be a complete Alexandrov space. The width at $p \in M, w_{p}$, is

Received by the editors September 29, 1992 and, in revised form, December 15, 1992.

1991 Mathematics Subject Classification. Primary 53C20.

Key words and phrases. Betti number, critical point. 
defined as

$$
w_{p}=\sup _{r \geq 0} \operatorname{diam}_{M}\left(S_{p}(r)\right)
$$

where $\operatorname{diam}_{M}\left(S_{p}(r)\right)$ denotes the diameter of the geodesic sphere $S_{p}(r)$ of radius $r$ around $p$, i.e., $\operatorname{diam}_{M}\left(S_{p}(r)\right)=\sup _{x, y \in S_{p}(r)} d(x, y)$. Define

$$
w(M)=\inf _{p \in M} w_{p}
$$

this is called the width of $M$.

He proved the following:

Theorem 1. Let $M$ be an $n$-dimensional complete Riemannian manifold with sectional curvature $K_{M} \geq k$ for some $k \leq 0$. Suppose that the width of $M$ satisfies $\sqrt{-k} w(M)<\frac{1}{2}$. Then the sum of the Betti numbers of $M$ satisfies

$$
\sum_{k=0}^{n} b_{k}(M) \leq C(n),
$$

where $C(n)>1$ is a constant depending only on $n=\operatorname{dim} M$.

We study a class of Alexandrov spaces and obtain an upper estimate for the Betti numbers in that class and in turn generalize Gromov's and Shen's theorems. More precisely, we prove

Main Theorem. Let $M$ be an n-dimensional complete Alexandrov space with sectional curvature $K_{M} \geq k$ for some constant $k \leq 0$. Suppose there is a point $p \in M$ and a number $D>0$ such that each connected component $K_{i}$ outside the ball $B_{p}(D \sqrt{-k})$ has the property that $\sqrt{-k} w_{p}^{\prime}<\frac{1}{2}$, where

$$
w_{p}^{\prime}=\sup _{r \geq D \sqrt{-k}} \operatorname{diam}_{K_{i}}\left(S_{p}(r)\right) .
$$

Then the sum of the Betti numbers of $M$ satisfies

$$
\sum_{k=0}^{n} b_{k}(M) \leq C(n, k, D)
$$

with respect to a field $F$, where $C(n, k, D)$ is a constant depending only on $k, n=\operatorname{dim} M$ and $D$.

Observe that the constant $C$ does not depend on the number of connected components outside $B_{p}(D \sqrt{-k})$. Also, under the assumption of our Main Theorem, the manifold has finite topological type; i.e., it is homeomorphic to the interior of a compact manifold with boundary.

For simplicity we assume that the condition in the Main Theorem is $K_{M} \geq$ -1 and there exists a point $p$ such that each connected component outside the ball $B_{p}(D)$ satisfies $w_{p}^{\prime}<\frac{1}{2}$. We further assume that $D>10$.

Before we prove our Main Theorem, let us state some basic lemmas.

\section{BASIC IDEAS AND LEMMAS}

The important concepts in Gromov's proof are critical point theory and the idea of content. 
1. Content. For two subsets $X \subset Y$ in a Riemannian manifold, Gromov [2] defined the content as the rank of the inclusion homomorphism.

$$
i_{*}: H_{k}(X, F) \hookrightarrow H_{k}(Y, F)
$$

written $b_{k}(X, Y)$. It is easy to see that, for subsets $X \subset \tilde{X} \subset \tilde{Y} \subset Y$ in $M$, $b_{k}(X, Y) \leq b_{k}(\tilde{X}, \tilde{Y})$.

He used the following important upper estimate for Betti numbers in his proof.

Theorem 2. Let $M$ be an n-dimensional complete Riemannian manifold with sectional curvature $K_{M} \geq-1$. Then there is a constant $C(n)>1$ depending only on $n$ such that for any $0<\varepsilon \leq 1$ and any bounded subset $X \subset M$

$$
\sum_{k=0}^{n} b_{k}\left(X, T_{\varepsilon} X\right) \leq\left(1+\frac{\operatorname{diam}_{M}(X)}{\varepsilon}\right)^{n} C(n)^{1+\operatorname{diam}_{M}(X)},
$$

where $\operatorname{diam}_{M}(X)$ denotes the diameter of $X$ in $M$ and $T_{\varepsilon} X$ denotes the $\varepsilon$ neighbourhood of $X$ in $M$.

Proof. For a detailed proof, refer to [1, pp. 478].

This result is generalized to Alexandrov spaces [4].

2. Critical point theory. Let $X$ be an Alexandrov space. For a triangle $\Delta x y z$ in $X$, let $\Delta \hat{x} \hat{y} \hat{z}$ be a triangle in the space form of curvature $k$ with $|\hat{x} \hat{y}|=|x y|$, $|\hat{y} \hat{z}|=|y z|$, and $|\hat{z} \hat{x}|=|z x|$. Let $\hat{L} x y z$ denote the angle at $\hat{y}$. Fix $p \in X$; a point $q \in X$ is called a critical point of $d(p, \cdot)$ if, for any $x \neq q, \hat{L} x q p \leq \frac{\pi}{2}$. A point $q$ is called regular if it is not critical. For a regular point $q$, there exists a point $x \neq q$ such that $\hat{L} x q p>\frac{\pi}{2}$. The result in [5] about regular maps implies that the following celebrated lemma generalizes to Alexandrov spaces.

Lemma 1 (Isotopy Lemma). Let $p \in X$. Suppose $\overline{B_{r_{2}}(p)} \backslash B_{r_{1}}(p)$ is free of critical points of $d_{p}$. Then $\overline{B_{r_{2}}(p)} \backslash B_{r_{1}}(p)$ is homeomorphic to $S_{r_{1}}(p) \times\left[r_{1}, r_{2}\right]$.

Hereafter, when it is clear in the context, we will use $x, y, z$ for $\hat{x}, \hat{y}, \hat{z}$ and $\angle x y z$ for $\hat{L} x y z$.

Lemma 2. Let $M$ be an Alexandrov space satisfying the above condition. Then, for each connected component $K_{i}$ outside the ball $B_{p}(D),\left(B_{p}(d(p, q)) \cap K_{i}\right) \backslash$ $\left(B_{p}(D) \cup B_{q}(10)\right)$ contains no critical point with respect to $p$ and $q$, respectively, where $q$ is any point in $K_{i}$. In particular, if $K_{i}$ is open, then $K_{i}$ contains no critical point with respect to $p$.

Proof. The idea of the proof is to apply Toponogov's Theorem [9] to a thin triangle. Assume on the contrary that, for some point $q \in K_{i}$ with $d(p, q) \geq$ $D,\left(B_{p}(d(p, q)) \cap K_{i}\right) \backslash\left(B_{p}(D) \cup B_{q}(10)\right)$ contains a critical point, say $x$, with respect to $p$ and $q$, respectively. Then there are two minimal geodesic segments $\sigma(t)$ and $\tau(t)$ issuing from $x$ to $p$ and $q$, respectively, such that $\hat{L} p x q \leq \frac{\pi}{2}$ makes an angle at most $\frac{\pi}{2} \cdot d(p, x) \leq d(p, q)$, and

$$
d(q, x)-d(x, \gamma(d(p, x))) \leq d(q, \gamma(d(p, x)))=d(p, q)-d(p, x) .
$$

Thus

$$
\begin{aligned}
d(p, x)+d(q, x) & =d(p, q)+d(x, \gamma(d(p, x))) \\
& \leq d(p, q)+\operatorname{diam}_{K_{i}}\left(\partial B_{p}(d(p, x))\right) \\
& \leq d(p, q)+w_{p}^{\prime} \leq d(p, q)+\frac{1}{2} .
\end{aligned}
$$


It is also easy to see that

$$
d(p, x)-d(q, x)=d(p, x)+d(q, x)-2 d(q, x) \leq d(p, q)-10
$$

and

$$
d(q, x)-d(p, x) \leq d(p, q)-10 .
$$

Applying Toponogov's Theorem to the triangle formed by $\sigma, \tau, \gamma$, we conclude that

$$
\cosh d(p, q) \leq \cosh d(p, x) \cosh d(q, x) .
$$

Thus

$$
\begin{aligned}
2 \leq & 4 e^{-d(p, q)} \cosh d(p, q) \leq 4 e^{-d(p, q)} \cosh d(p, x) \cosh d(q, x) \\
= & e^{d(p, x)+d(q, x)-d(p, q)}+e^{-d(p, x)+d(q, x)-d(p, q)} \\
& +e^{d(p, x)-d(q, x)-d(p, q)}+e^{-d(p, x)-d(q, x)-d(p, q)} \\
\leq & e^{1 / 2}+3 \varepsilon^{-10}<2,
\end{aligned}
$$

which is a contradiction.

Lemma 3. Let $X_{1}, X_{2}$ be subsets of a topological space. Then

$$
b_{q}\left(X_{1} \cup X_{2}\right) \leq b_{q}\left(X_{1}\right)+b_{q}\left(X_{2}\right)+b_{q-1}\left(X_{1} \cap X_{2}\right) .
$$

Proof. From the Mayer-Vietoris sequence and its exactness,

$$
\begin{aligned}
\cdots & \rightarrow H_{q+1}\left(X_{1} \cup X_{2}\right) \rightarrow H_{q}\left(X_{1} \cap X_{2}\right) \rightarrow H_{q}\left(X_{1}\right) \oplus H_{q}\left(X_{2}\right) \\
& \rightarrow H_{q}\left(X_{1} \cup X_{2}\right) \rightarrow H_{q-1}\left(X_{1} \cap X_{2}\right) \rightarrow \cdots .
\end{aligned}
$$

\section{Proof of Main Theorem}

Suppose $M$ contains some open connected component $K_{i}$ outside the geodesic ball $B_{p}(D)$. Lemma 2 shows that $K_{i} \backslash B_{p}(D)$ does not contain critical points with respect to $p$. By the Isotopy Lemma, there is an isotopy of $M$ which sends $K_{i}$ into $B_{p}(D)$, and we obtain a subset $M^{\prime}$ of $M$ that does not contain $K_{i}, M^{\prime} \subset M \backslash K_{i} \subset M$. Thus

$$
\sum_{k=0}^{n} b_{k}(M) \leq \sum_{k=0}^{n} b_{k}\left(M \backslash K_{i}, M\right) \leq \sum_{k=0}^{n} b_{k}\left(M \backslash K_{i}\right),
$$

so, without loss of generality, we want to assume that $M$ does not contain an open connected component outside $B_{p}(D)$.

Claim. The number of bounded connected components outside $B_{p}(R D)$ in this class of Alexandrov spaces is uniformly bounded if $R=R(n, D)$ is chosen to be sufficiently large.

Proof of Claim. Let $q_{i}$ and $q_{j}$ be two critical points in $K_{i}$ and $K_{j}$, respectively. Any geodesic between $q_{i}$ and $q_{j}$ has to go into the $B_{p}(D)$ because $K_{i}$ and $K_{j}$ are disconnected components outside $B_{p}(D)$. Let $d\left(p, q_{i}\right)=b D$ and $d\left(p, q_{j}\right)=c D$, so $d\left(q_{i}, q_{j}\right) \geq(b-1) D+(c-1) D$. We assume here that $b, c \geq 2$ without loss of generality. We find a corresponding triangle having the same length in the hyperbolic space of constant curvature -1 . Let the angle opposite the geodesic $q_{i} q_{j}$ be $\alpha$ and the corresponding angle in the hyperbolic space be $\bar{\alpha}$. 
The corresponding triangle in the hyperbolic space has to satisfy

$$
\cosh ((b-1) D+(c-1) D)=\cosh b D \cosh c D-\sinh b D \sinh c D \cos \bar{\alpha} .
$$

But $\alpha \geq \bar{\alpha}$ by Topogonov's Theorem. Thus $\cos \alpha \leq \cos \bar{\alpha}$, so

$$
\begin{aligned}
\cos \alpha \leq & \frac{\cosh b D \cosh c D-\cosh (b+c-2) D}{\sinh b D \sinh c D}, \\
\cos \alpha \leq & \operatorname{coth} b D \operatorname{coth} c D-\frac{\cosh (b-1) D \cosh (c-1) D}{\sinh b D \sinh c D} \\
& -\frac{\sinh (b-1) D \sinh (c-1) D}{\sinh b D \sinh c D} .
\end{aligned}
$$

However,

$$
\begin{gathered}
\lim _{b \rightarrow \infty} \operatorname{coth} b D=1, \\
\lim _{b \rightarrow \infty} \frac{\cosh (b-1) D}{\sinh b D}=\lim _{b \rightarrow \infty} \frac{e^{(b-1) D}+e^{-(b-1) D}}{e^{b D}-e^{-b D}}=\lim _{b \rightarrow \infty} \frac{e^{(2 b-1) D}+e^{D}}{e^{2 b D}-1}=e^{-D}, \\
\lim _{b \rightarrow \infty} \frac{\sinh (b-1) D}{\sinh b D}=\lim _{b \rightarrow \infty} \frac{e^{(b-1) D}+e^{-(b-1) D}}{e^{b D}-e^{-b D}}=e^{-D} .
\end{gathered}
$$

Thus

$$
\lim _{b, c \rightarrow \infty} \cos \alpha \leq 1-2 e^{-2 D}<1
$$

i.e., the angle $\alpha$ is bounded telow by a constant strictly bigger than 0 . Let $\Sigma_{p}$ denote the space of directions at $p$ and $\xi_{i} \in \Sigma_{p}$ denote the direction of the minimal segment $p q_{i}$. The above argument shows that there is a sufficiently large $R=R(n, D)$ such that $\left|\xi_{i} \xi_{j}\right| \geq \varepsilon(n, D)>0, i \neq j$. Since $\Sigma_{p}$ is an $(n-1)$-dimensional Alexandrov space with curvature $\geq 1$ and diam $\leq 1$ [9], using the comparison theorem in [4], one can conclude that the number $m$ of such $K_{i}$ is bounded from above by $N=N(n, D)$. This completes the proof of the claim.

Let $q$ be a point in $K_{i}$, a bounded connected component outside $B_{p}(D)$, such that $l_{p}:=\sup _{x \in K_{i}} d(p, x)=d(p, q)$. We assume first that $l_{p} \geq R D$, $R>1$, as in the claim.

Let $X_{i 1}=K_{i}$ and $X_{i 2}=M \backslash B_{q}(10)$.

$X_{i 1}$ is homotopy equivalent to $B_{q}(10)$ by Lemma 2 , so we have

$$
\sum_{k=0}^{n} b_{k}\left(X_{i 1}\right) \leq \sum_{k=0}^{n} b_{k}\left(B_{q}(10), B_{q}(11)\right) \leq \sum_{k=0}^{n} b_{k}\left(B_{q}(10), T_{1}\left(B_{p}(10)\right)\right) .
$$

The second expression is bounded by a constant depending only on $n$.

There exists an isotopy which sends $B_{p}(R D) \cup K_{i} \backslash B_{q}(10)$ into $B_{p}(R D)$. Thus $X_{i 2}$ is homotopy equivalent to a subset of $M \backslash K_{i}$, say $X_{i 3}$.

On the other hand, $X_{i 1} \cap X_{i 2}$ is homotopy equivalent to a compact subset $X_{i 4}$, by the flow of the integral curves

$$
\sum_{k=0}^{n} b_{k}\left(X_{i 1} \cap X_{i 2}\right) \leq \sum_{k=0}^{n} b_{k}\left(X_{i 4}, T_{1} X_{i 4}\right) \leq\left(1+\operatorname{diam}_{M}\left(X_{i 4}\right)\right)^{n} C(n)^{1+\operatorname{diam}_{M}\left(X_{i 4}\right)},
$$

since $\operatorname{diam}_{M}\left(X_{i 4}\right) \leq 2 \operatorname{width}_{p}\left(X_{i 4}\right)<1$. Thus $\sum_{k=0}^{n} b_{k}\left(X_{i 4}\right)$ is bounded by a constant depending only on $n$. 
We can then obtain the upper bound for the sum of Betti numbers of $M$ provided we obtain the upper bound for the sum of Betti numbers of $X_{i 2}$, which is the same as that of $X_{i 3}$. To do this, we just take another bounded connected component outside $B_{p}(D)$, say $K_{j}$, where $i \neq j$, and we repeat the procedure used for $K_{i}$. We see that we will obtain the upper bound for the sum of Betti numbers of $X_{i 3}$ provided we obtain that of $X_{j 3}$. We keep repeating this process until we get a compact subset $Y$ that does not contain any connected component with $l_{p}>R D$ outside $B_{p}(R D)$. It is clear that we will only have to repeat that process for at most $m$ times since there are at most $m$ such components by our claim. In conclusion, we will have the upper bound for the sum of Betti numbers for $M$ provided we have that of $Y$. However, we already know that the sum of Betti numbers for $Y$ is bounded by

$$
\sum_{k=0}^{n} b_{k}(Y)=\sum_{k=0}^{n} b_{k}\left(Y, T_{1} Y\right) \leq \sum_{k=0}^{n} b_{k}(R D,(R+1) D),
$$

which depends only on $n$ and $D$.

Thus, combining all this, we obtain explicitly a bound for $M$ and complete our proof.

\section{REFERENCES}

1. U. Abresch, Lower curvature bounds, Topologov's theorem, and bounded topology 2, Ann. Sci. École Norm. Sup. (4) 20 (1987), 475-502.

2. M. Gromov, Curvature, diameter and Betti numbers, Comment. Math. Helv. 56 (1981), 179-195.

3. _ـ Structures métriques pour les variétés Riemanniennes, Cedic Fernand-Nathan, Paris, 1981.

4. Z. Liu and Z. Shen, On the Betti numbers of Alexandrov spaces, preprint.

5. G. Perelman, Alexandrov's spaces with curvature bounded from below, II, preprint.

6. J. Sha and D. Yang, Examples of manifolds of positive Ricci curvature, J. Differential Geom. 29 (1989), 95-103.

7. 33 (1991), 127-138.

8. Z. Shen, Finite topological type and vanishing theorems for Riemannian manifolds, Ph.D. Thesis, SUNY Stony Brook.

9. M. Gromov, Y. Burago, and G. Perelman, Alexandrov's spaces with curvature bounded from below, preprint.

Department of Mathematics, National University of Singapore, Kent Ridge, SingaPORE 0511, SINGAPORE 